\title{
Multi-Objective Optimization of Transonic Compressor Blade Using Evolutionary Algorithm
}

\author{
Yongsheng Lian* \\ University of Michigan, Ann Arbor, Michigan 48109 \\ and \\ Meng-Sing Liou ${ }^{\dagger}$ \\ NASA John H. Glenn Research Center at Lewis Field, Cleveland, Ohio 44135
}

\begin{abstract}
Multi-objective optimization is performed of the NASA rotor67 transonic compressor blade. The objectives are to maximize the stage pressure ratio, as well as to minimize the compressor weight. The backbones of the optimization approach consist of a genetic algorithm, a gradient-based method, and a response surface model. The genetic algorithm is used to facilitate the multi-objective optimization and to find the global optima of highdimensional problems. The gradient-based method accelerates the optimization convergence rate. The response surface model, constructed to replace the computationally expensive analysis tool, reduces the computational cost. Representative solutions are selected from the Pareto-optimal front to verify against the computational fluid dynamics tool. Compared with the baseline design, some optimal solutions increase the stage pressure ratio by $1.8 \%$ and decrease the weight by $5.4 \%$. A detailed study of flow structure near peak efficiency is presented by means of pressure distribution and streamlines inside boundary layers. Results show that the optimized blade favors a lighter weight by a thinner blade shape. The stage pressure rise is attributed to a reduced separation zone and a weakened shock wave.
\end{abstract}

\section{Introduction}

$\mathbf{N}$ ASA is working at reducing carbon dioxides, nitrogen oxides, and aerosols from the nation's airspace, specifically those gases generated by gas turbine engines used in commercial aircraft. NASA's goals are to reduce landing and takeoff nitrogen oxides by $70 \%$ of the 1996 international civil aviation organization standard and to reduce carbon dioxide by $15 \%$ from current state-of-the-art large engines, both by 2007 . Light weight, compact, and efficient fans are essential to achieve these goals.

Today, axial flow fans have been developed to a point where stage efficiency has exceeded $90 \%$. Further improvement is not readily achievable. Therefore, the goal of the current research and development is to reduce the engine size and weight without sacrificing its efficiency. ${ }^{1}$ The compressor, which tends to have many stages to achieve an optimum operating pressure ratio, is a fairly massive part of the engine. Reduction in size can be achieved by reduced diameter, reduced length, or both.

Unfortunately, these approaches either have limited gains or suffer from mechanical and aeroelastic problems. This leaves increased pressure ratio per stage as the most promising technique of achieving more compact compressors. ${ }^{1}$ If considerable improvement is achieved on stage pressure ratio, we may be able to reduce the number of stages required for an aircraft engine. This would consequently reduce the number of parts in the manufacturing and maintenance inventory, as well as the size and weight in the surrounding aircraft structure that support the engine, leading to even greater weight savings.

Many researchers have used optimization techniques to improve the engine compressor performance. Among them, to name a few,

Received 27 November 2004; revision received 16 April 2005; accepted for publication 16 April 2005. Copyright (C) 2005 by the American Institute of Aeronautics and Astronautics, Inc. The U.S. Government has a royaltyfree license to exercise all rights under the copyright claimed herein for Governmental purposes. All other rights are reserved by the copyright owner. Copies of this paper may be made for personal or internal use, on condition that the copier pay the $\$ 10.00$ per-copy fee to the Copyright Clearance Center, Inc., 222 Rosewood Drive, Danvers, MA 01923; include the code 0748-4658/05 \$10.00 in correspondence with the CCC.

*Visiting Scholar, Department of Aerospace Engineering, 1320 Beal Avenue; ylian@umich.edu. Member AIAA.

${ }^{\dagger}$ Senior Scientist, Mail Stop 5-11, Department of Aerospace Engineering. Associate Fellow AIAA.
Oyama et al. minimized the entropy generation of the NASA rotor67 blade, ${ }^{2}$ later, Lian and Liou performed multi-objective optimization to increase the stage pressure ratio and to decrease the entropy generation of the same blade, ${ }^{3}$ Benini performed multi-objective optimization to improve the total pressure ratio and adiabatic efficiency of the NASA rotor 37 blade, ${ }^{4}$ and Mengistu and Ghaly conducted multipoint optimization to improve compressor performance over the full range of operating condition. ${ }^{5}$ All of these works used evolutionary algorithms (EAs) as the optimizer. There are several underlying reasons for the popularity of EAs. First, EAs are easy to implement. Typically an EA code has about 1000 lines with FORTRAN or C language. Second, EAs can be easily coupled with other analysis tools because optimization with EAs does not require gradient information. Third, EAs have the capability to find the global optima. Another thrust is that EAs are particularly suitable for multi-objective optimization problems, which are often encountered in real design problems and have raised growing interest among researchers from different disciplines. However, EAs suffer a slow convergence rate because they use probabilistic recombination operators to control the step size and searching direction. When computationally expensive analysis tools are used, the required CPU time impedes their practical applications even today.

To tackle this challenge, we hybridize a genetic algorithm (GA) with a response surface model to provide an efficient and robust approach aimed at solving computationally expensive design optimization problems. ${ }^{3}$ The rationale is to take the advantage of GAs in multi-objective optimization and response surface models in computational efficiency. In addition, we perform local search after the GA optimization to speed up the optimization convergence rate.

In this work we aim at performing multi-objective optimization for the NASA rotor67 compressor blade. Our objectives are to maximize the stage pressure ratio and to minimize the compressor weight. This paper is structured as follows: We first formulate our problem; then we briefly introduces our computational fluid dynamics (CFD) tool; this is followed by introduction of the key elements used in the optimization approach, including a response surface model, a genetic algorithm, and a gradient-based method; last, we present our optimization results. Detailed comparisons are made between the NASA rotor67 and one optimal design by means of blade shape, pressure distribution, and flow patterns inside the boundary layer. 


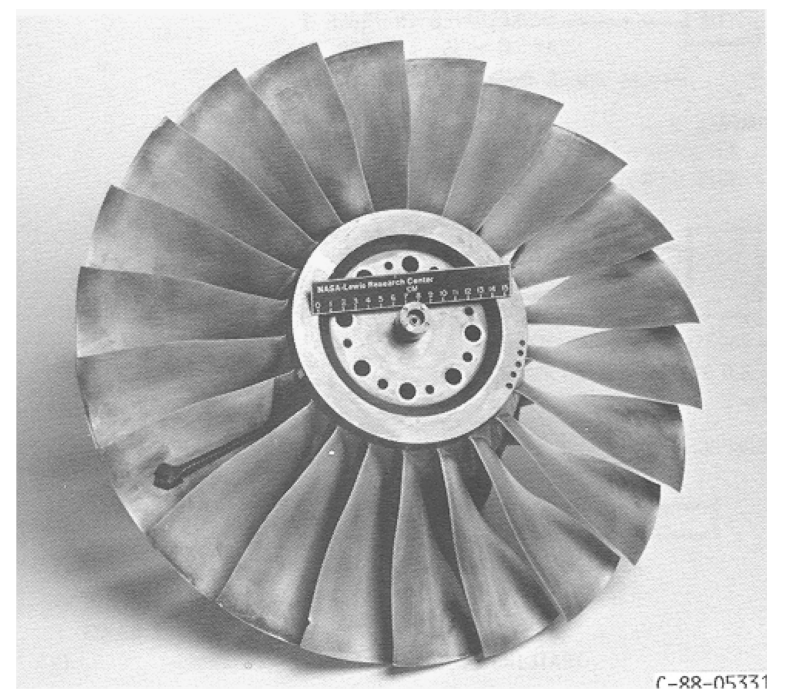

Fig. 1 Test rotor.

\section{Problem Formulation}

What we consider here is how to redesign the NASA rotor67 compressor blade. ${ }^{6}$ The rotor67 is a transonic axial-flow fan rotor and is the first stage of a two-stage fan. The test rotor with 22 blades is shown in Fig. 1. The inlet and exit tip diameters are 51.4 and $48.5 \mathrm{~cm}$, respectively. The inlet and exit hub/tip radius ratios are 0.375 and 0.478 , respectively. Based on average span/root axial chord, the rotor has a low aspect ratio of 1.56. The solidity, defined as the ratio of chord length to spacing between two adjacent blades, varies from 3.11 at the hub to 1.29 at the tip. At the design point, it has a stage pressure ratio of 1.63 at a mass flow rate of $33.25 \mathrm{~kg} / \mathrm{s}$. The design rotational speed is $16,043 \mathrm{rpm}$, which generates a tip speed of $429 \mathrm{~m} / \mathrm{s}$ and a relative inlet tip Mach number of 1.38 . The Reynolds number based on the chord length at the hub and the inlet velocity is $1.797 \times 10^{6}$. The blade is made of generic titanium (Ti-6Al-4V), which has a density of $4510 \mathrm{~kg} / \mathrm{m}^{3}$.

Our problem can be formulated as the following: Maximize $p_{02}(\boldsymbol{x}) / p_{01}(\boldsymbol{x})$ and minimize $W(\boldsymbol{x})$ subject to $\left|\dot{m}-\dot{m}_{\mathrm{bl}}\right| / \dot{m}_{\mathrm{bl}}<0.5 \%$, where $\boldsymbol{x}_{L} \leq \boldsymbol{x} \leq \boldsymbol{x}_{U}$ and where $p_{02}$ is the outlet total pressure, $p_{01}$ is the inlet total pressure, and $p_{02} / p_{01}$ is the stage pressure ratio. We use $\dot{m}$ to represent the mass flow rate and use subscript bl to denote the baseline design. The mass flow rate and the mass-averaged total pressure are computed with the CFD tool; the blade weight $W$ is computed by integrating the blade volume. Vector $x$ represents the design variable, and $\boldsymbol{x}_{L}$ and $\boldsymbol{x}_{U}$ are the lower and upper bounds of the design variables, respectively. The lower and upper bounds are defined as 0.95 and 1.05 of the baseline value, respectively.

The aerodynamic objective is to maximize the stage pressure ratio, whereas the structural objective is to minimize the blade weight. The aerodynamic constraint is imposed to ensure the new design has comparable mass flow rate as the baseline design. Note that the two objectives are competing: Improving one objective will deteriorate the other. Instead of having one single optimal solution, we expect a set of compromised solutions among which a solution is better than the others in terms of one objective but is worse in terms of the other. These compromised solutions are usually called Paretooptimal solutions in the context of multi-objective optimization. The curve formed by joining these solutions is known as a Pareto-optimal front.

The designed blade geometry is defined by superimposing a perturbation blade on the baseline rotor67 blade. The perturbation blade is obtained by linear interpolation of four airfoil profiles along the span (hub, 31\% span, $62 \%$ span, and tip). Each airfoil profile can be defined by a mean camber line and thickness distributions and is parameterized by a third-order B-spline curve. The camber is determined by three design variables (with the leading- and trailing-edge points fixed) and the thickness distribution by five design variables. As a result, eight design variables are required to represent such a perturbation airfoil profile, resulting in 32 design variables in total. By doing this, we can readily recover the baseline geometry by setting all of the design variables to zero. To make the optimization results comparable to those of the baseline configuration, we maintain the chord distribution along the span and fix the meridional contours of the hub, casing, sweep, and lean.

\section{CFD Code}

A high-fidelity CFD tool, TRAF3D, is used to evaluate the objective functions and constraints. ${ }^{7,8}$ TRAF3D solves the following three-dimensional, unsteady, Reynolds-averaged Navier-Stokes equations for a rotating blade passage in conservative form in the curvilinear coordinate:

$$
\frac{\partial \boldsymbol{Q}}{\partial t}+\frac{\partial \boldsymbol{F}}{\partial \xi}+\frac{\partial \boldsymbol{G}}{\partial \eta}+\frac{\partial \boldsymbol{H}}{\partial \zeta}=\frac{\partial \boldsymbol{F}_{v}}{\partial \xi}+\frac{\partial \boldsymbol{G}_{v}}{\partial \eta}+\frac{\partial \boldsymbol{H}_{v}}{\partial \zeta}+\boldsymbol{S}
$$

The conservative variable vector and fluxes are

$$
\begin{gathered}
\boldsymbol{Q}=J^{-1}\left\{\begin{array}{c}
\rho \\
\rho u \\
\rho v \\
\rho w \\
\rho e
\end{array}\right\}, \quad \boldsymbol{F}=J^{-1}\left\{\begin{array}{c}
\rho U \\
\rho u U+\xi_{x} p \\
\rho v U+\xi_{y} p \\
\rho w U+\xi_{z} p \\
\rho h U+\xi_{t} p
\end{array}\right\} \\
\boldsymbol{G}=J^{-1}\left\{\begin{array}{c}
\rho V \\
\rho u V+\eta_{x} p \\
\rho v V+\eta_{y} p \\
\rho w V+\eta_{z} p \\
\rho h V+\eta_{t} p
\end{array}\right\}, \quad \boldsymbol{H}=J^{-1}\left\{\begin{array}{c}
\rho W \\
\rho u W+\zeta_{x} p \\
\rho v W+\zeta_{y} p \\
\rho w W+\zeta_{z} p \\
\rho h W+\zeta_{t} p
\end{array}\right\} \\
\boldsymbol{S}=J^{-1}\left\{\begin{array}{c}
\rho \Omega w \\
0 \\
0 \\
-\rho \Omega v \\
0
\end{array}\right\}
\end{gathered}
$$

where $u, v, w, p, T, e$, and $h$ are the density; velocity components in the $x, y$, and $z$ directions; pressure; temperature; specific total energy; and specific total enthalpy, respectively. $\Omega$ is the angular velocity of the rotating Cartesian system $x, y, z . J$ is the Jacobian of transformation. $U, V$, and $W$ are the contravariant velocities. The viscous terms are $\boldsymbol{F}_{v}, \boldsymbol{G}_{v}$, and $\boldsymbol{H}_{v}$. The space discretization uses a second-order cell-centered scheme with eigenvalue scaling to weigh the artificial dissipation terms. The system of equations is advanced in time using an explicit four-stage Runge-Kutta scheme. The twolayer eddy-viscosity model of Baldwin and Lomax is used for the turbulence closure.

Standard boundary conditions for subsonic flows are implemented. At the inlet, the flow angles, total pressure, and total temperature are specified at the inlet, whereas the magnitude of the velocity is taken from the interior. At the subsonic-axial outlet, the average value of the static pressure at the hub is prescribed, whereas circumference pressure gradient is extrapolated to maintain a specified average static pressure. The density and velocity components are extrapolated from interior. The radial pressure equilibrium assumption is applied in the calculations. On the solid wall, the temperature is set constant as the total temperature at the inlet and the pressure is extrapolated from the interior. The no-slip boundary conditions and the temperature condition are used together to compute the density and total energy. Periodic boundary conditions are applied from blade to blade passage.

The tip clearance region is handled by the periodic condition across the blade without any modeling of the region. When the tip clearance region is included in the computation, it is generally done with an additional patch of grid. This will present a difficulty in the design procedure because the shape is changing with design and grid generation will have to be done in an automatic fashion, which is 


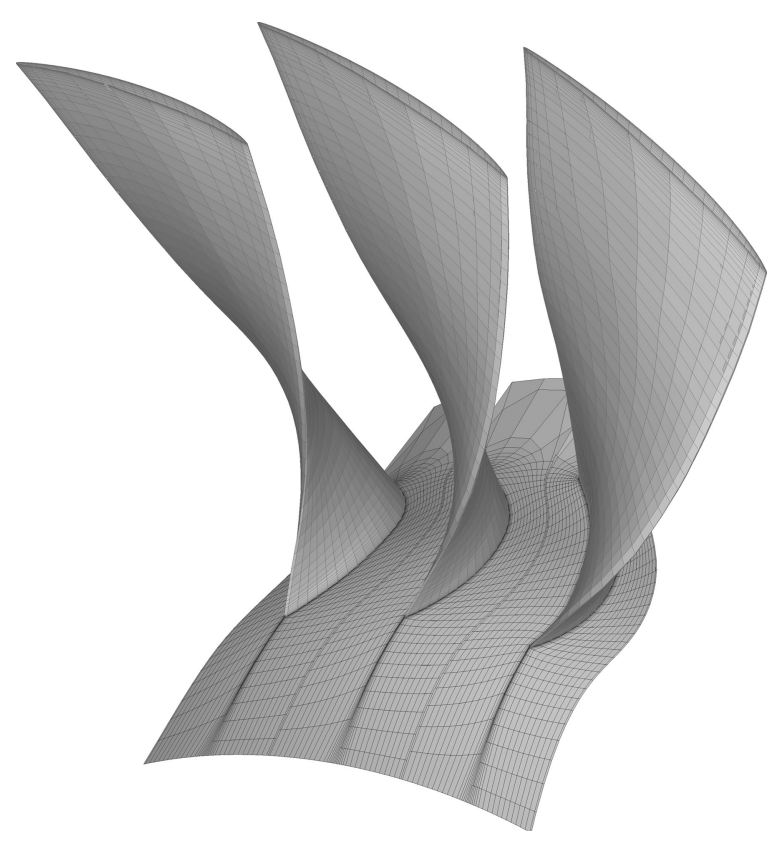

Fig. 2 Structured grid with $6.0 \times 10^{5}$ nodes per passage.

difficult when there are multiple meshes involved. In addition, for the rotor67, it is found that the tip clearance effect becomes important only when the stall condition is approached. ${ }^{8}$ Because the present design is focused on the near-maximum efficiency condition, the tip clearance effort is not significant. Therefore, it is considered to be a good representation of the flow without gridding up the tip region.

For a single passage, the chordwise, tangential, and spanwise grid point numbers are 201,53 , and 57 , respectively. In total, the structured grid has $6.0 \times 10^{5}$ nodes per single passage as shown in Fig. 2.

The code has been applied in the study of both cascade flows ${ }^{7}$ and rotor flows. ${ }^{8}$ Its predictive capability is validated against the experimental data and other codes. In the study of rotor67 blade, the computed performance map and radial distributions of the static pressure, total pressure, and total temperature match the experimental data ${ }^{6}$ and the calculation of Chima. ${ }^{9}$ Figure 3 a shows the computational result of the relative Mach number contours at the $90 \%$ span from the hub. The shock position and strength match well with the experimental result in Fig. 3b.

\section{Optimization Approach}

The adopted optimization method aims at providing an efficient and robust way to solve computationally expensive and multiobjective optimization problems. The backbones of this method include a response surface model, a GA, and a gradient-based method. To make it standalone, we briefly introduce each key element.

Response surface models are built to approximate computationally expensive functions. They are usually orders of magnitude cheaper than the original problems but still provide good approximation. The response surface model is normally chosen to be a low-order polynomial. The second-order polynomial is widely used due to its flexibility and ease of use. A second-order response surface model with $d$ design variables can be written as follows:

$$
y=\beta_{0}+\sum_{i=1}^{d} \beta_{i} x_{i}+\sum_{i=1}^{d} \beta_{i i} x_{i}^{2}+\sum_{j=2}^{d} \sum_{i=1}^{j-1} \beta_{i j} x_{i} x_{j}+\varepsilon
$$

where $y$ can be the objective or constraint functions, $x_{i}$ is the design variable, $\beta$ is the unknown coefficient, and $\varepsilon$ is the total error, which is the difference between the observed value $y$ and the approximated value with the polynomial.

Constructing the response surface is to find the unknown coefficients, and it has the following steps. First, we sample a number of distinct design points $\left\{x_{i k}\right\}_{k=1}^{p}$, where $P$ is the number of design

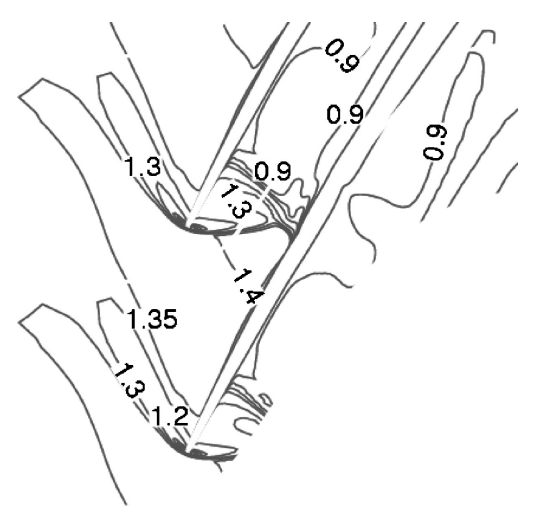

a) Numerical result

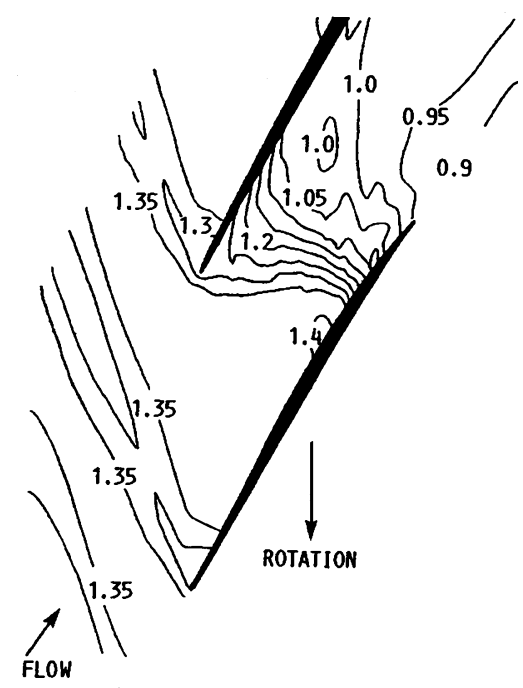

b) Experimental result

Fig. 3 Comparison of Mach number contours at $90 \%$ span from hub.

points and usually larger than the number of coefficients. Second, we evaluate these design points using the CFD tool to obtained the observed values $\left\{y_{k}\right\}_{k=1}^{p}$. Third, we substitute the design points and the observed values into Eq. (3) and obtain the following linear system of equations:

$$
\begin{array}{r}
y_{k}=\beta_{0 k}+\sum_{i=1}^{d} \beta_{i k} x_{i k}+\sum_{i=1}^{d} \beta_{i i k} x_{i k}^{2}+\sum_{j=2}^{d} \sum_{i=1}^{j-1} \beta_{i j k} x_{i k} x_{j k}+\varepsilon_{k} \\
k=1, \ldots, p
\end{array}
$$

The second-order response surface model of Eq. (3) contains $(d+1)(d+2) / 2$ coefficients, and we need to sample at least that many distinct design points to determine the unknown coefficients. When the number of equations is larger than the number of unknown coefficients, we have an overdetermined problem. At last, we use the method of least squares to determine the unknown coefficients. In the current context we use the least squares to minimize the sum of the squares error, $\varepsilon_{k}$.

When deterministic analysis models such as the CFD tools are used, a good experimental design tends to fill the design space rather than to concentrate on the boundary. In our work, we apply the improved hypercube sampling algorithm ${ }^{10}$ to sample the design points. This method tends to spread out the sampling points as evenly as possible by determining an optimal even spacing. Lian and Liou found that a $20-80 \%$ overdetermined design for the second-order response surface model gave reasonably good results. ${ }^{3}$

We employ GAs as our optimizer. GAs are motivated by the principles of natural genetics and natural selection. ${ }^{11}$ Evolutionary theory tells us that in nature those species who better adopt the environment survive and mate to produce offsprings. The offsprings 
inherit good genes from their parents and potentially have better adaptivity than their parents. Occasionally, the offsprings may alter themselves by gene mutation. In parallel to the natural selection, GAs have three fundamental operators: reproduction, crossover, and mutation. Simply put, the reproduction operator chooses good solutions and eliminates bad solutions; the crossover operator recombines good solutions and hopefully creates better new solutions; the mutation operator changes the solutions locally for potentially better solutions.

GAs are simple and straightforward. It is suitable for multiobjective optimization problems. In dealing with multi-objective optimization problems, gradient-based methods usually transform a multi-objective problem into a single-objective problem by introducing weight functions, which usually favor a particular Paretooptimal solution. On the other hand, GA's population approach can be exploited to emphasize equally all Pareto-optimal solutions in a population and to preserve a diverse set of multiple Pareto-optimal solutions. As a consequence, GAs can find as many Pareto-optimal solutions as possible in one run. This eliminates the need to convert a multi-objective optimization problem into multiple single-objective optimization problems by introducing new parameters that favor certain Pareto-optimal solutions.

Because GAs use probabilistic combination operators literally to control the step size and searching direction, their convergence rate is usually slow when optimal solutions are approached. Many researchers have hybridized GAs with local search methods, typically gradient-based methods, to speed up the convergence rate. ${ }^{12-15}$ Even though their implementations are different, the underlying idea is to switch to local search whenever the GA convergence rate slows down. We employ commercially available software Design Optimization Tools (DOT) ${ }^{16}$ as our local search tool. DOT solves both unconstraint and constraint problems using gradient-based methods. In solving our constraint problem, we choose the sequential quadratic programming method from DOT. Gradient information is obtained by finite difference. In using DOT, we keep the blade weight as the objective and convert the pressure ratio as one constraint. The new problem, therefore, is a bounded optimization problem with two constraints.

There is no strict rule on when the GA optimization should terminate and the gradient-based optimization should begin. In practice, it is proper to switch to the gradient-based optimization when the GA optimization convergence rate slows down. Usually solutions from GA optimization are taken as the starting points for the local search. This approach takes the advantages of both GAs and gradient-based methods and can improve the search efficiency and ensure the global search ability simultaneously.

In conclusion, the optimization approach has the following steps:

1) Sample distinct design points using the improved hypercube sampling.

2) Evaluate the design points using the CFD code.

3) Construct the response surface models.

4) Apply the GA on the response surface models and to search for the Pareto-optimal solutions.

5) Switch to local search once the GA optimization convergence rate slows down.

6) Choose representative solutions and verify them against the CFD code.

\section{Numerical Results}

There are 32 design variables in our problem and 561 unknown coefficients in Eq. (3). We sample 1024 design points, which represent an $80 \%$ overdetermined design. These design points are evaluated using the TRAF3D code to give the mass flow rate and pressure ratio. The blade weight is computed by integrating the blade volume. Three response surface models are thereafter constructed: two for the objective functions and one for the constraint. The accuracy of these models is evaluated by statistical measures, including the coefficient of determination, $R^{2}$, the adjusted coefficient of determination, $R_{\mathrm{adj}}^{2}$, and the percentage of root mean square error (\%RMSE), which is the ratio of root mean square error to the mean of response. The coefficient of determination, $R^{2}$, measures the proportion of the
Table 1 Statistical measures of quadratic response surface approximations

\begin{tabular}{lccc}
\hline \hline Error statistics & $p_{02} / p_{01}$ & $W$ & $\dot{m}$ \\
\hline$R^{2}$ & 0.9949 & 0.9999 & 0.9979 \\
$R_{\text {adj }}^{2}$ & 0.9888 & 0.9999 & 0.9954 \\
$\%$ RMSE & $0.3000 e-3$ & $0.1175 e-3$ & $0.1270 e-3$ \\
\hline \hline
\end{tabular}

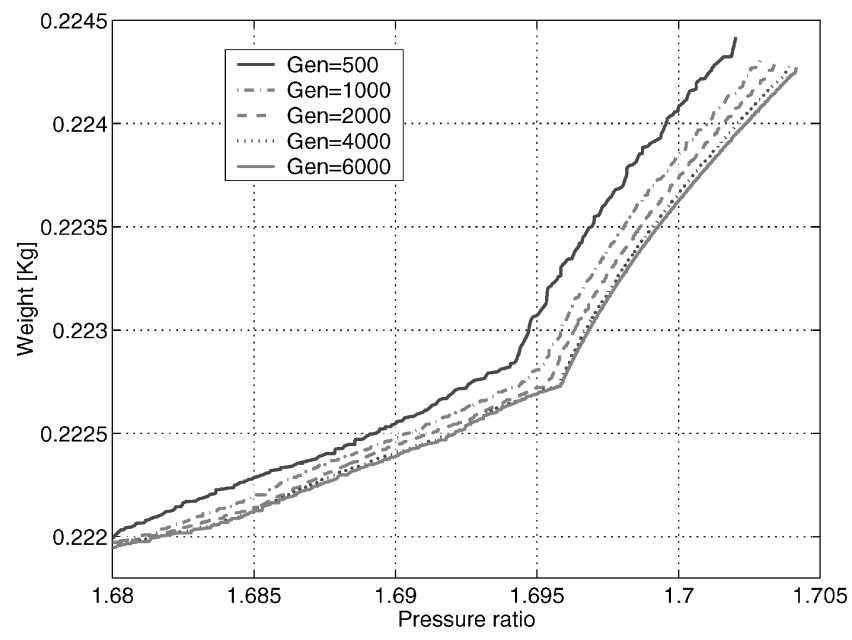

Fig. 4 Convergence history of GA.

variation around the mean accounted by the model. It has a range between 0 and 1 . A value of 1 means a perfect fit with zero errors. The adjusted coefficient of determination is more comparable over models with different numbers of parameters by using the degrees of freedom in its computation. Unlike $R^{2}$, which always decreases if extra terms are added to the model, $R_{\text {adj }}^{2}$ decreases when redundant terms are added. Table 1 shows the test result. The value of $R_{\text {adj }}^{2}$ for the stage pressure rise has a value of $R_{\text {adj }}^{2}$ larger than 0.98 , indicating that the response surface model has a good representation of the variability observed from the design points; likewise, the values of $R_{\text {adj }}^{2}$ for the weight function and constraint function also suggest that the quadratic response approximations have good predictive capabilities.

The population size of the GA is set as 320 . Figure 4 shows the convergence history. The optimization result clearly improves as more generations are continued. The convergence rate is fast at the early stage and becomes slow later on. Numerical test shows that an increase in the generation size can improve the convergence further. However, an increase in the generation size is not always a panacea because it will not only increase the computational cost but also raise another question on when to terminate the GA optimization.

A frequently used remedy is to hybridize a GA with a gradientbased method. We switch to a gradient-based method after the GA optimization. The gradient-based method is the sequential quadratic programming method from commercial software DOT. The starting points of the gradient-based optimization are the Pareto-optimal solutions from the GA optimization at the 6000th generation. The gradient-based method does improve the solution, and the improved Pareto-optimal front is shown in Fig. 5. In terms of computational cost, the gradient-based optimization evaluates each response surface for approximately 16,000 times, which is equivalent to the number of functions evaluated by GA in 50 generations. Figure 5 shows that the improved Pareto-optimal front is better than that from GA optimization using 20,000 generations, even though the improvement may be minor.

The improved Pareto-optimal front has 473 Pareto-optimal solutions. It is time consuming to evaluate all of them. Here we use the K-means cluster algorithm ${ }^{17}$ to choose 16 representative solutions to verify with the CFD code. The validated results together with the baseline design are shown in Fig. 6. Compared with the baseline design, all of the solutions increase the stage pressure ratio 
Table 2 Rotor67 design performance

\begin{tabular}{lcccc}
\hline \hline Design & $\begin{array}{c}\text { Mass flow, } \\
\mathrm{kg} / \mathrm{s}\end{array}$ & $\begin{array}{c}\text { Isentropic } \\
\text { efficiency }\end{array}$ & $\begin{array}{c}\text { Pressure } \\
\text { ratio }\end{array}$ & $\begin{array}{c}\text { Weight } \\
\text { ratio }\end{array}$ \\
\hline Minimal volume & 33.682 & 0.9166 & 1.669 & 0.9464 \\
Maximal pressure ratio & 33.355 & 0.9153 & 1.697 & 0.9552 \\
Rotor67 & 33.446 & 0.9123 & 1.666 & 1.000 \\
\hline \hline
\end{tabular}

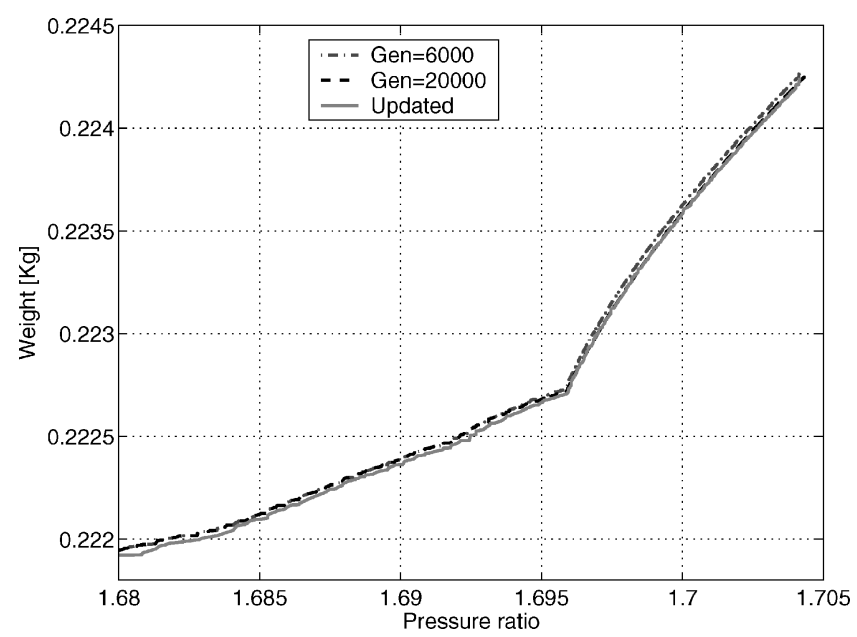

Fig. 5 Updated Pareto-optimal front using local search.

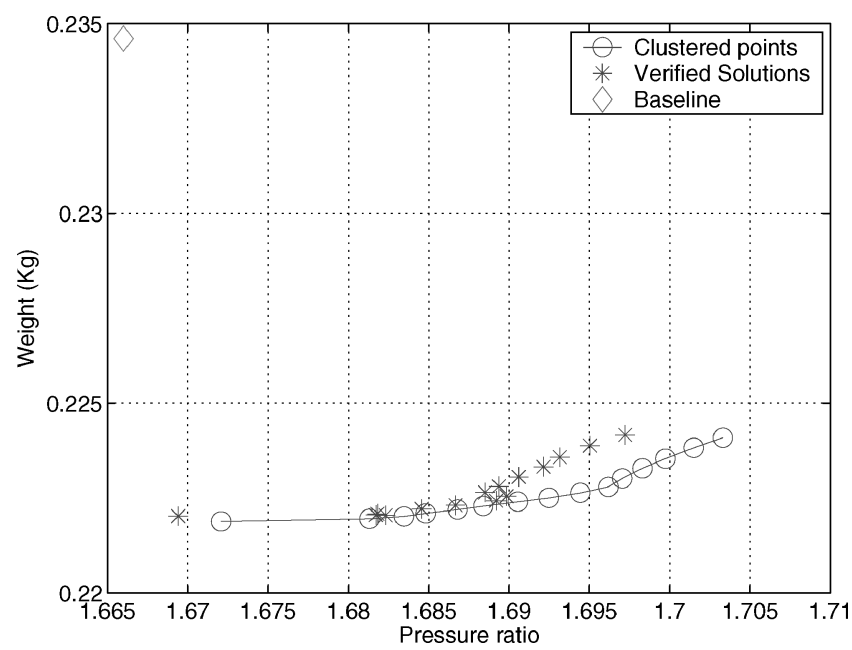

Fig. 6 Pareto-front optimal solutions for rotor67.

and reduce the weight. Some Pareto-optimal solutions increase the pressure ratio by as much as $1.8 \%$ and reduce the weight by $5.4 \%$.

Among the 16 verified solutions, we choose two designs representing the design with minimal weight and maximal pressure ratio, respectively. We compare their aerodynamic performance with the baseline design. In Table 2 we list their performance characteristics, including the mass flow rate and the isentropic efficiency. The minimal weight design has a larger mass flow rate and higher isentropic efficiency than the baseline, whereas the high pressure ratio design has a slightly smaller mass flow rate but higher isentropic efficiency than the baseline.

All of the new designs in Fig. 6 have a larger pressure ratio and lighter weight than the rotor67. The design with the maximal pressure ratio will be more meaningful in explaining the efforts of shape change on pressure distribution and flow pattern. Therefore, in this paper, we compare the pressure distribution and flow pattern between the baseline and the maximal pressure ratio design. Three spanwise blade profiles are shown in Figs. 7-9, representing the span of 10,50 , and $90 \%$, respectively. The static pressure coefficient profiles are also shown. At the $10 \%$ span, the high-pressure

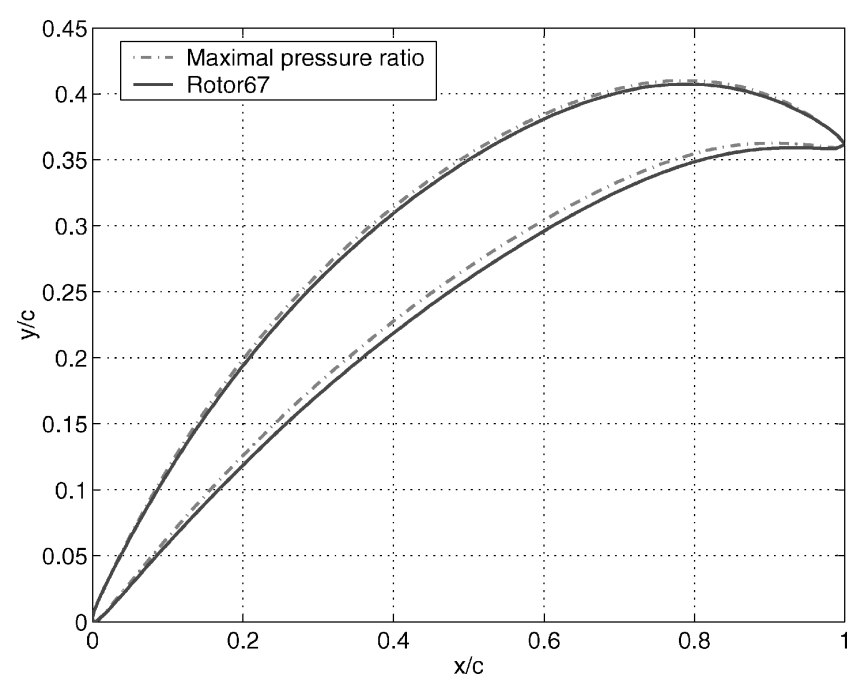

a) Airfoil shape

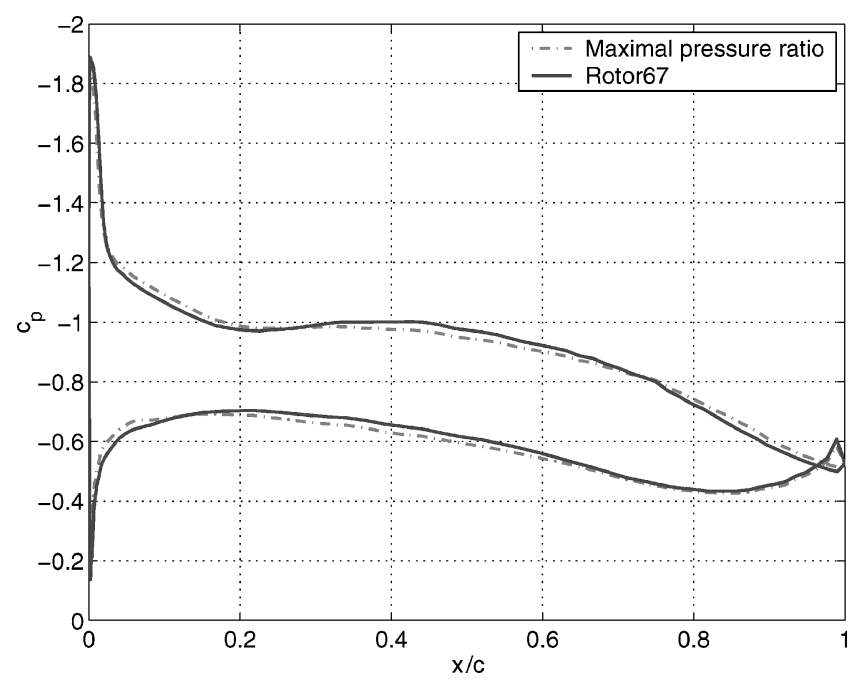

b) Pressure distribution

Fig. 7 Comparison between maximal pressure ratio design and baseline at $10 \%$ span from hub.

ratio design has a larger camber but less thickness than the rotor67 design. The thinner airfoil contributes to the lighter weight of the new design. The difference in the pressure distribution is rather small. The same conclusion can be made at the $50 \%$ span. At the $90 \%$ span, the high-pressure ratio design has a slightly smaller camber and thinner airfoil than the rotor67. Nevertheless, the pressure difference is rather large, indicating that transonic flow is sensitive to the shape change. One noticeable change is the shock position. The new design has a more forward shock wave than the baseline.

Figures 10-12 present the relative Mach number contours at three spanwise positions, again, at the 10,50 , and $90 \%$ span, respectively. At the $10 \%$ span, the two contours are similar, except the supersonic bubble is weaker in the new design than in the baseline; the Mach number is 1.21 and 1.3 , respectively. At the $50 \%$ span, an oblique shock stands ahead of the blade. The two shocks have comparable strength. At the $90 \%$ span, the shock system has a lambda structure with a bow shock. The relative Mach number of the maximal pressure design is 1.48 , which is weaker than 1.52 in the baseline. The weaker shock is helpful to decrease the global losses.

We use the pressure distribution on the suction side to interpret the flow pattern. Figure 13 shows a comparison of the difference between the maximal pressure design and the rotor67 design. Both designs have a strong passage shock in the upper part of the rotor. In the central part of the blade span, the passage shock loses its intensity and becomes weak. In comparison to Fig. 12, the maximal pressure design has a more forward shock than the baseline. This 


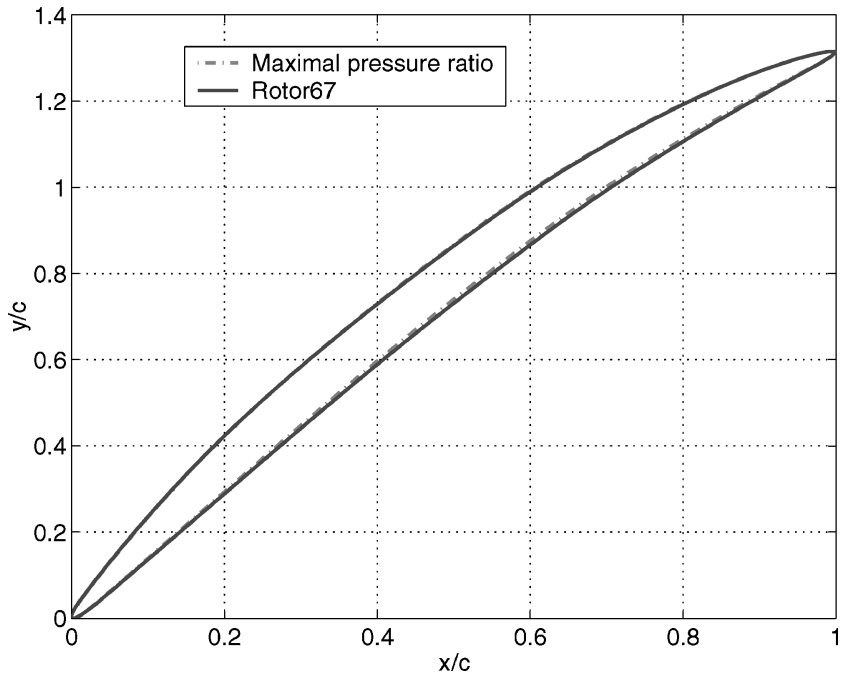

a) Airfoil shape

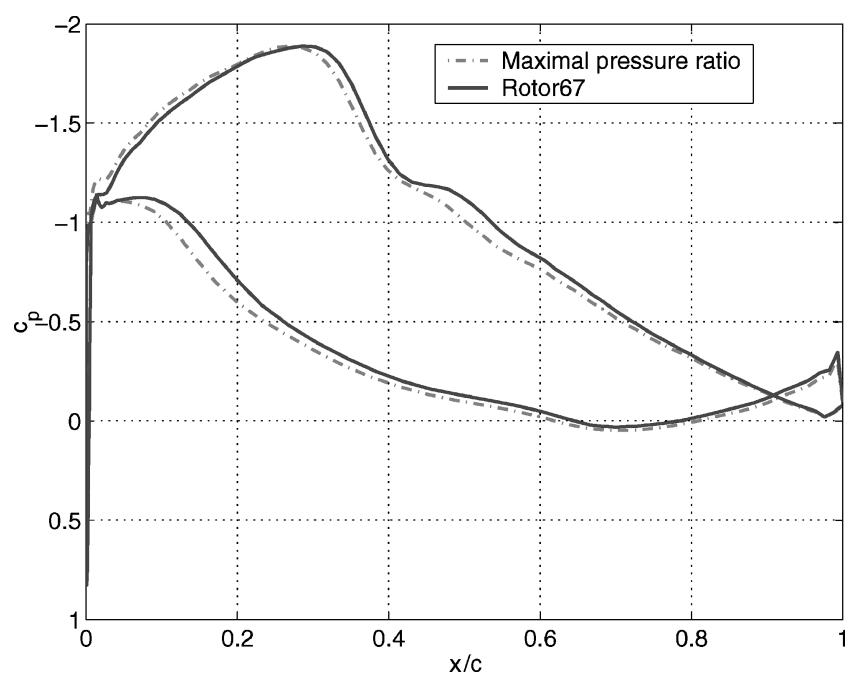

b) Pressure distribution

Fig. 8 Comparison between maximal pressure ratio design and baseline at $50 \%$ span from hub.

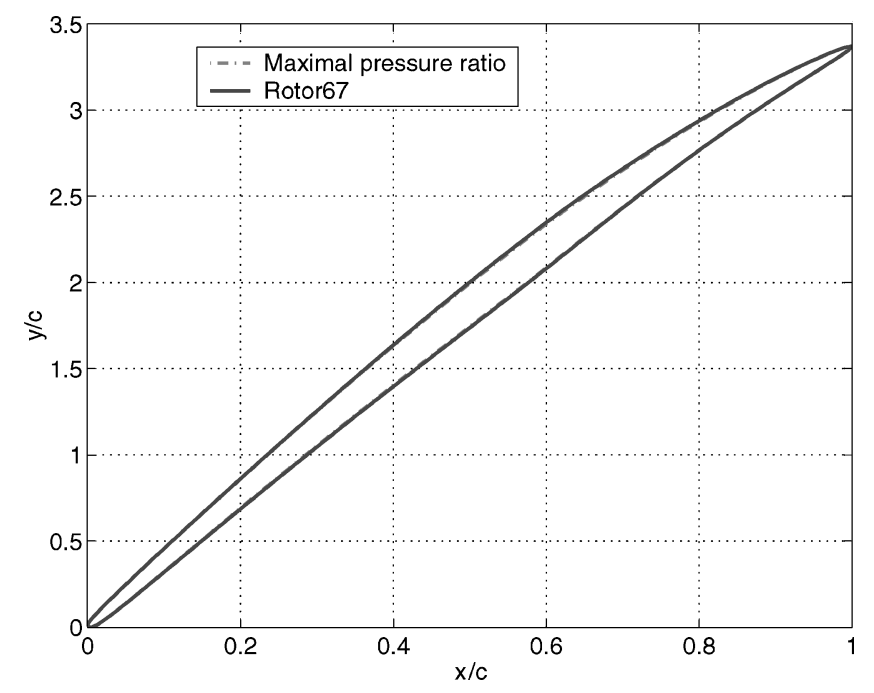

a) Airfoil shape

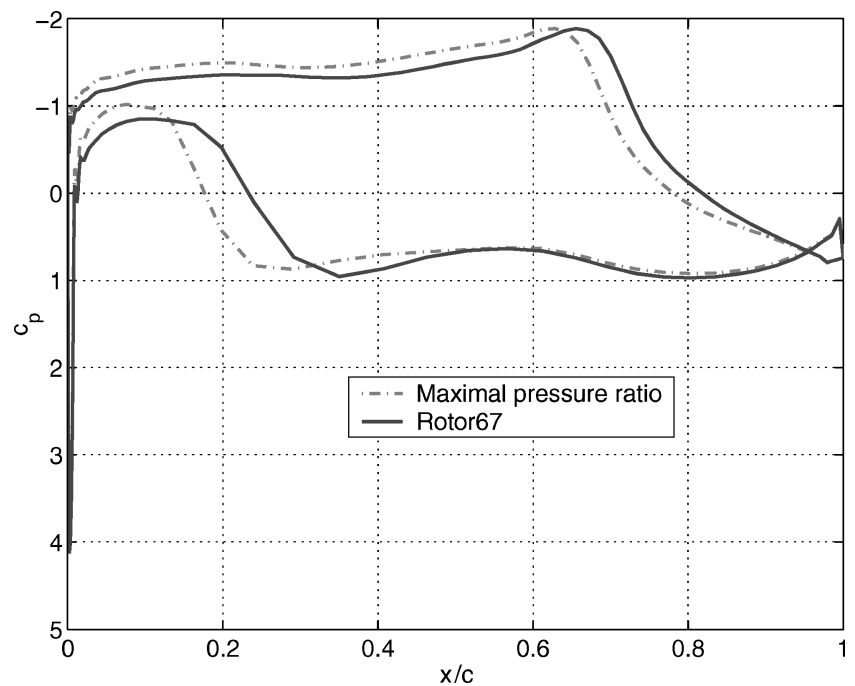

b) Pressure distribution

Fig. 9 Comparison between maximal pressure ratio design and baseline at $90 \%$ span from hub.

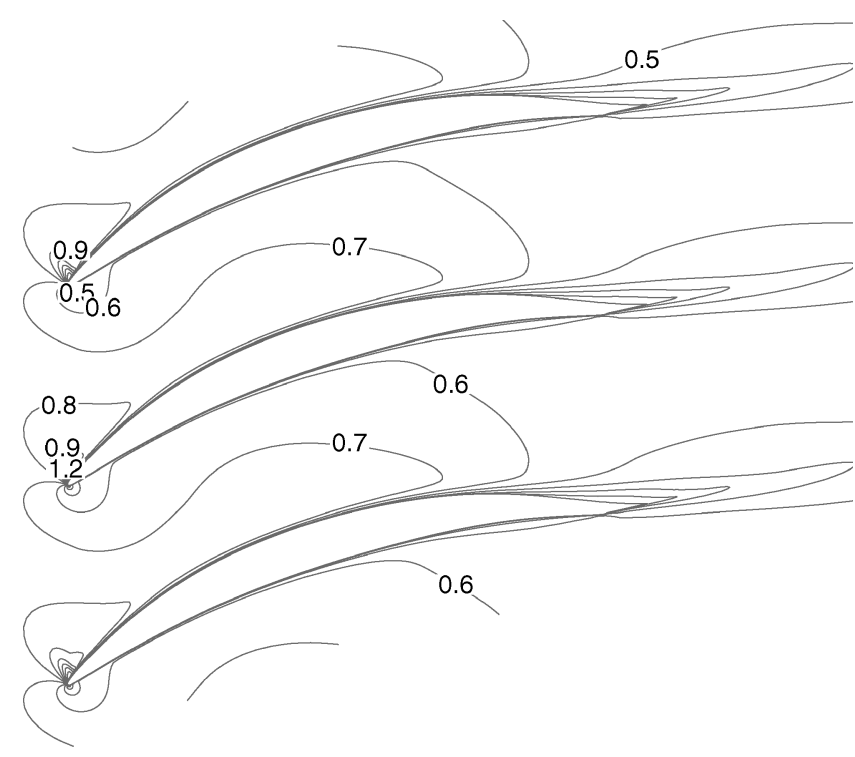

a) Rotor67

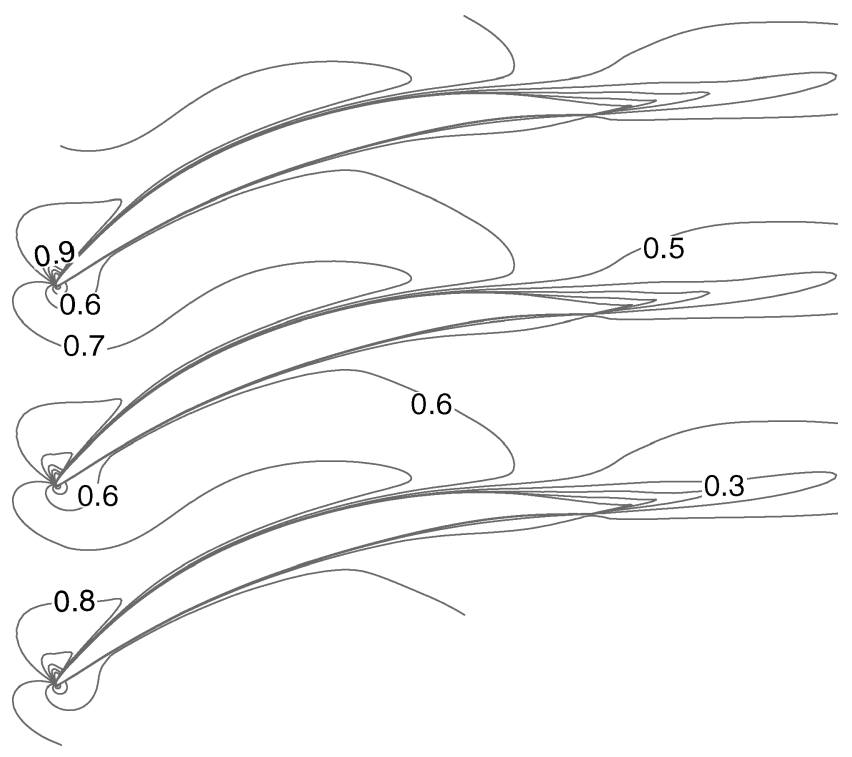

b) High-pressure ratio design

Fig. 10 Relative Mach number contours at $10 \%$ span. 


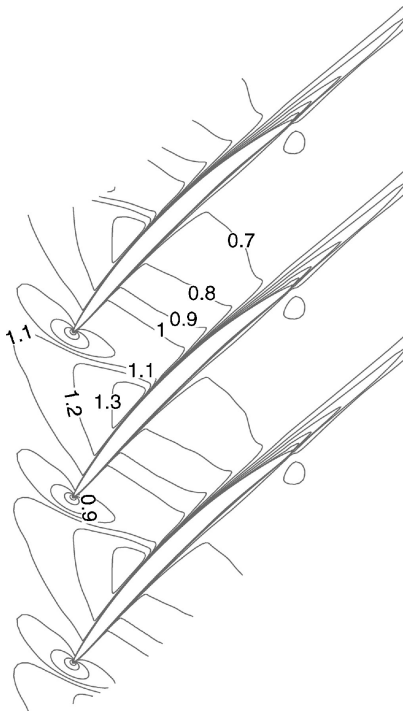

a) Rotor67

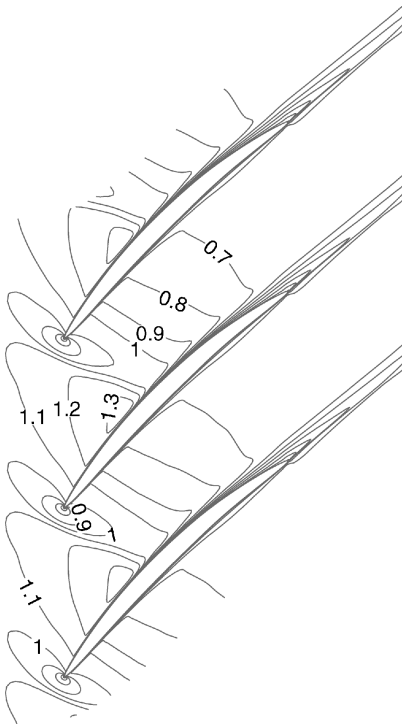

b) High-pressure ratio design

Fig. 11 Relative Mach number contours at 50\% span.

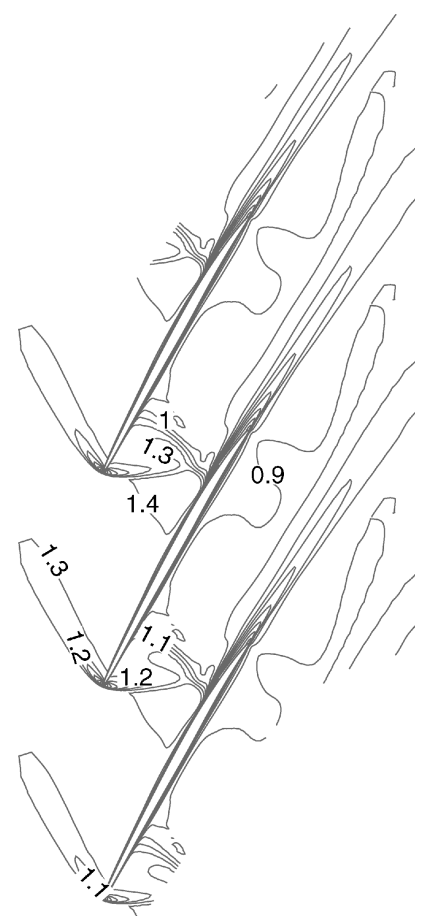

a) Rotor67

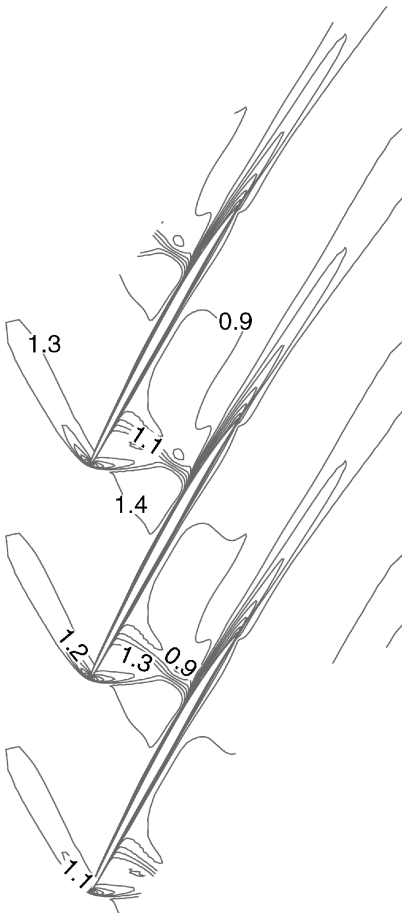

b) High-pressure ratio design

Fig. 12 Relative Mach number contours at $90 \%$ span.

observation is consistent with our finding from the pressure distribution in Fig. 9. After the shock, flow separates as a result of shockboundary-layer interaction. This separation is evident in Fig. 14. Here, separation lines are characterized by flows going toward the line, whereas reattachment lines look like flows are going away from the line. Compared with the rotor67, after the shock the maximal pressure design has a smaller separation zone, which is partially responsible for its higher stage pressure ratio. The trailing-edge enlargement shows the flow never reattaches after separation for the baseline. These separation zones are also evident in the streamwise velocity contours in Fig.15, where the separation zone is indicated with a negative velocity. Also notice that in the leading edge close to the hub the low momentum fluid separates and moves radially outward before it turns in the streamwise direction. Also, close to the hub, because of the adverse pressure gradient, flow liftoff occurs and eventually flow separation happens near the trailing edge.

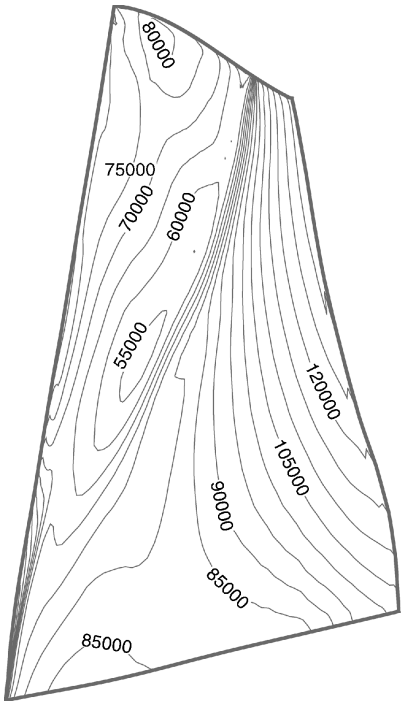

a) Rotor67

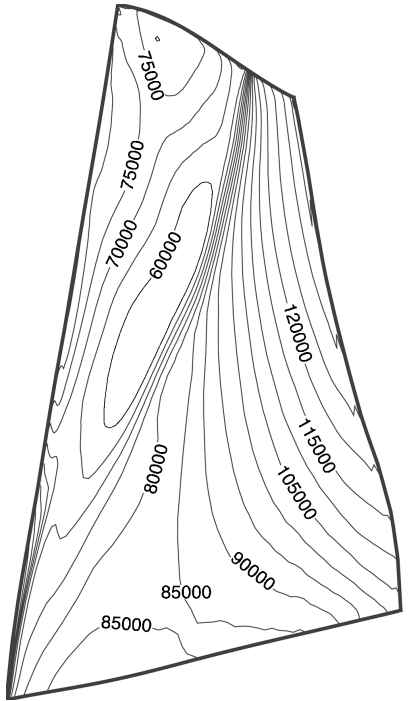

b) High-pressure ratio design
Fig. 13 Pressure contours on blade suction side.

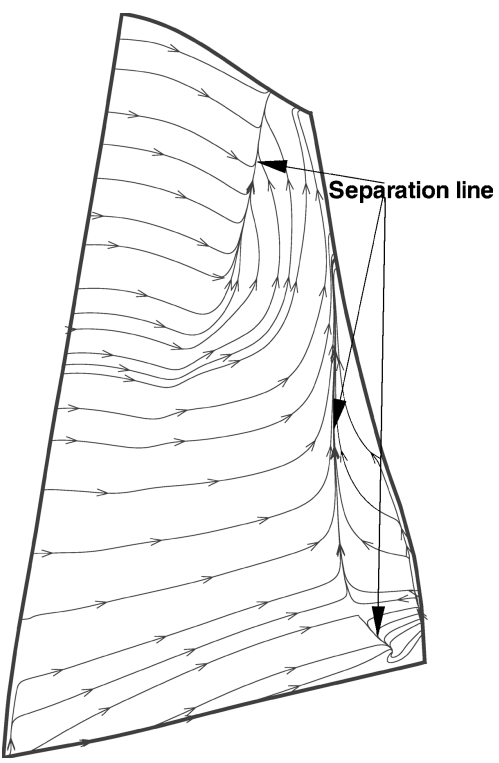

a) Rotor67

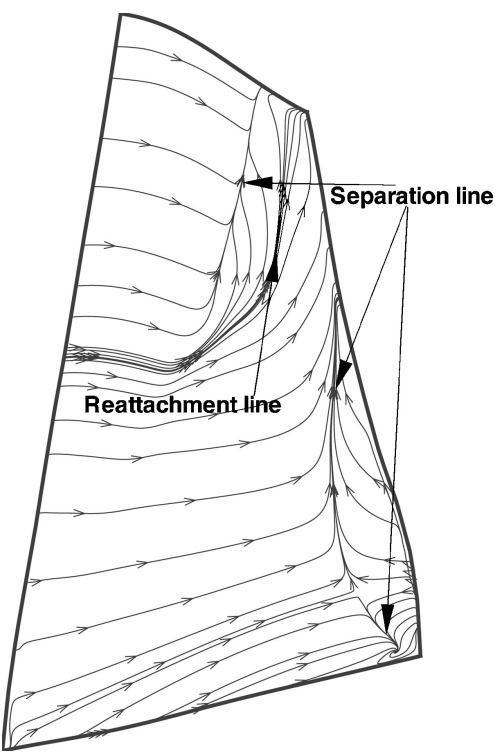

b) High-pressure ratio design

Fig. 14 Streamlines close to blade suction side. 


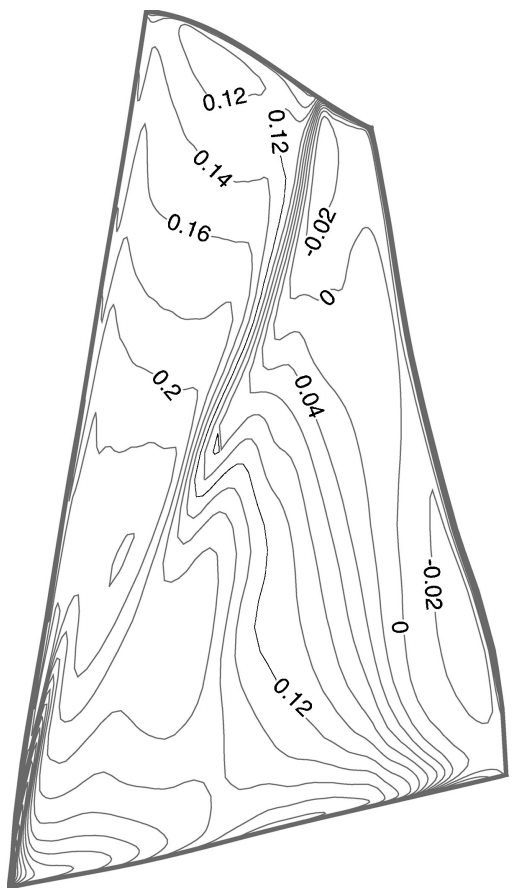

a) Rotor67

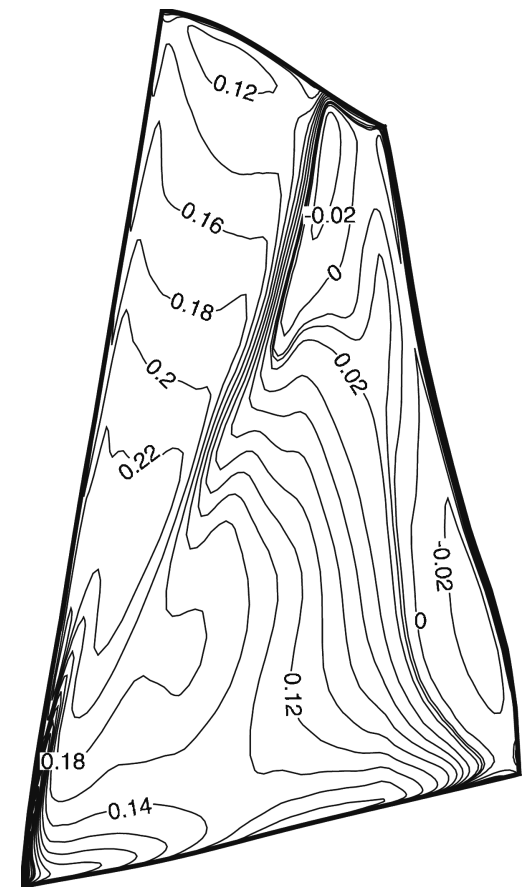

b) High-pressure ratio design

Fig. 15 Streamwise velocity contours close to blade suction side.

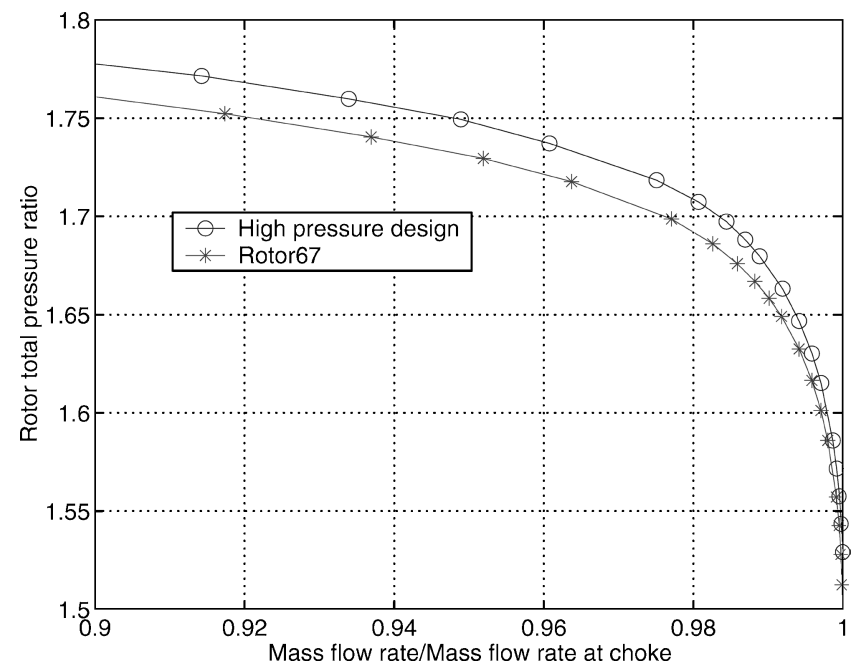

a) Pressure ratio

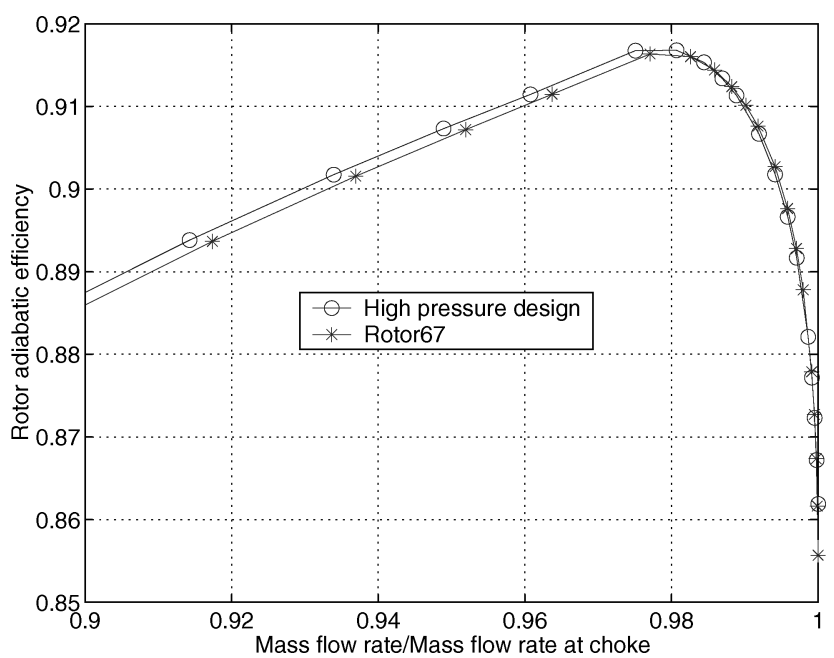

b) Adiabatic efficiency

Fig. 16 Compressor blade design speed operating characteristics.

Figure 16a shows the performance maps of both the baseline and the maximal pressure ratio design. At the operating condition, the new design has a higher total pressure ratio than the baseline. This observation is also true at off-design conditions. Figure $16 \mathrm{~b}$ shows that the new design has a higher adiabatic efficiency than the baseline at all of the operating conditions.

The turn-around time for this optimization is one week using eight Intel Itantium 2 1.3-GHz processors. With the increase of computational power, we expect the turn-around time can be reduced to $24 \mathrm{~h}$. Furthermore, to prove eventually that the current procedure works and that CFD is accurate enough to distinguish performance changes with these changes in geometry, we need to built and test the optimized geometry.

\section{Summary}

We performed multi-objective design optimization for the NASA rotor67 blade using a hybrid method. The backbones of this method include a GA and a quadratic response surface approximation.
We further implemented a gradient-based method after the GA to accelerate the convergence rate. With this method, we achieved an improvement of $1.8 \%$ in the pressure ratio and $5.4 \%$ decrease in weight for the rotor67 at a much reduced computational cost.

\section{Acknowledgment}

This work is supported by NASA Research Grant NAG3-2869 under the Ultra Efficient Engine Technology Program.

\section{References}

${ }^{1}$ Benser, W. A., "Transonic Compressor Technology Advancements" NASA SP-304, 1970.

${ }^{2}$ Oyama, A., Liou, M. S., and Obayashi, S., "Transonic Axial-Flow Blade Shape Optimization Using Evolutionary Algorithm and Three-Dimensional Navier-Stokes Solver,' Journal of Propulsion and Power, Vol. 20, No. 4, 2004, pp. 612-619. 
${ }^{3}$ Lian, Y., and Liou, M. S., "Multiobjective Optimization Using Coupled Response Surface Model and Evolutionary Algorithm," AIAA Journal (to be published); also AIAA Paper 2004-4323, 2004.

${ }^{4}$ Benini, E., "Three-Dimensional Multi-Objective Design Optimization of a Transonic Compressor Rotor," Journal of Propulsion and Power, Vol. 20, No. 3, 2004, pp. 559-565.

${ }^{5}$ Mengistu, T., and Ghaly, W., "Single and Multipoint Shape Optimization of Gas Turbine Blade Cascades," AIAA Paper 2004-4446, Aug. 2004.

${ }^{6}$ Strazisar, A. J., Wood, J. R., Hathaway, M. D., and Suder, K. L., "Laser Anemometer Measurements in a Transonic Axial-Flow Fan Rotor," NASA TP 2879, 1989.

${ }^{7}$ Arnone, A., Liou, M. S., and Povinelli, L. A., "Multigrid Calculation of Three-Dimensional Viscous Cascade Flows," NASA TM-105257, 1991.

${ }^{8}$ Arnone, A., "Viscous Analysis of Three-Dimensional Rotor Flow Using a Multigrid Method," Journal of Turbomachinery, Vol. 116, No. 3, 1994, pp. 435-445.

${ }^{9}$ Chima, R. V., "Viscous Three-Dimensional Calculations of Transonic Fan Performance," CP-510, AGARD, Feb. 1992, pp. 21-1-21-19.

${ }^{10}$ Beachkofski, B., and Grandhi, R., "Improved Distributed Hypercube Sampling," AIAA Paper 2002-1274, April 2002.
${ }^{11}$ Deb, K., Multi-Objective Optimization Using Evolutionary Algorithms, Wiley, New York, 2001.

${ }^{12}$ Muyl, F., Dumas, L., and Herbert, V., "Hybrid Method for Aerodynamic Shape Optimization in Automotive Industry," Computers and Fluids, Vol.33, No. 5-6, 2004, pp. 849-858.

${ }^{13}$ Ong, Y. S., Nair, P. B., and Keane, A., "Evolutionary Optimization of Computationally Expensive Problems via Surrogate Modeling," AIAA Journal, Vol. 41, No. 4, 2003, pp. 687-696.

${ }^{14}$ Lian, Y., Liou, M.-S., and Oyama, A., "An Enhanced Evolutionary Algorithm with a Surrogate Model," Proceeding of Genetic and Evolutionary Computation Conference-GECCO 2004 [CD-ROM], edited by Deb et al., Seattle, WA, Springer-Verlag, 2004.

${ }^{15}$ Goel, T., Vaidyanathan, R., Haftka, R. T., Queipo, N., Shyy, W., and Tucker, K., "Response Surface Approximation of Pareto Optimal Front in Multi-objective Optimization," AIAA Paper 2004-4501, Aug. 2004.

16“DOT User's Manual," Ver. 4.20, Vanderplaats Research \& Development, Inc., Colorado Springs, CO, 1995.

${ }^{17}$ Bishop, C. M., Neural Network for Pattern Recognition, Oxford Univ. Press, 2003, pp. 187-189. 